\title{
Title: Hydroxyurea Use among Children with Sickle Cell Anemia
}

Authors: Reeves, $\mathrm{SL}^{1,2}$; Jary, $\mathrm{HK}^{1}$; Gondhi, $\mathrm{JP}^{1}$; Raphael, JL ${ }^{3}$; Lisabeth, LD ${ }^{2,4}$; Dombkowski, $\mathrm{KJ}^{1}$

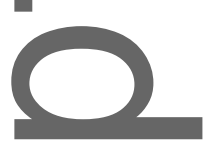

AuthorAffiliations: ${ }^{1}$ Susan B Meister Child Health Evaluation and Research Center,

Department of Pediatrics, University of Michigan, Ann Arbor, MI; ${ }^{2}$ Department of

Epidemiology, School of Public Health, University of Michigan, Ann Arbor, MI;

${ }^{3}$ Department of Pediatrics, Baylor College of Medicine, Houston, TX; ${ }^{4}$ Department of

Neurology, University of Michigan, Ann Arbor, MI

Corresponding author: Sarah Leasure Reeves, PhD, 300 North Ingalls, Rm 6D19 Ann Arbor,

MI 48109-5456; Phone: 734-615-8319; Fax: 734-232-1400; Email: sleasure@umich.edu

Word counts: Max 99 abstract, 1489 main text

1 table, 1 figure, $\theta$ supplemental files

Short running title: Hydroxyurea among Children with Sickle Cell Anemia

Keywords: sickle cell anemia, hydroxyurea, Medicaid, Administrative claims

Abbreviations key:

\begin{tabular}{|l|l|}
\hline Abbreviation & Full Term/Phrase \\
\hline SCA & Sickle Cell Anemia \\
\hline NHLBI & National Heart, Lung, and Blood Institute \\
\hline Hb & Hemoglobin \\
\hline NHLBI & National Heart, Lung, and Blood Institute \\
\hline
\end{tabular}

This is the author manuscript accepted for publication and has undergone full peer review but has not been through the copyediting, typesetting, pagination and proofreading process, which may lead to differences between this version and the Version of Record. Please cite this article as doi: 10.1002/pbc.27721.

This article is protected by copyright. All rights reserved. 


\begin{tabular}{|l|l|}
\hline MAX & Medicaid Analytic eXtract \\
\hline CMS & Centers for Medicare and Medicaid Services \\
\hline ED & Emergency Department \\
\hline ICD-9-CM & Modification \\
\hline NDC & National Drug Code \\
\hline SD & Standard Deviation \\
\hline
\end{tabular}

\section{Abstract}

This study describes hydroxyurea use among children ages 1-17 with sickle cell anemia (SCA) enrolled in at least one year of Medicaid in 6 states from 2005-2012. Administrative claims were used to summarize the number of days' supply of hydroxyurea dispensed by state and year. A total of 7,963 children with SCA contributed 22,424 person-years. Among person-years with at least 30 days of hydroxyurea, only 18\% received at least than 300 days of hydroxyurea, which varied by state. Following updated recommendations for all children with SCA to begin hydroxyurea, strategies to increase hydroxyurea adherence among this population-are needed.

\section{Introduction}

Sickle cell disease is a group of inherited red blood cell disorders predominately affecting minority populations in the US. ${ }^{1,2}$ There are numerous subtypes of sickle cell disease, the most severe of which is sickle cell anemia (SCA). SCA consists of the subtypes hemoglobin (Hb) SS and $\mathrm{Hb} \beta^{0}$ Thalassemia. ${ }^{2}$ Among children, SCA is associated with significant morbidity, the most common of which is recurrent pain. ${ }^{2,3}$ These pain crises 
negatively impact the quality of life, as well as pose a significant burden of cost and utilization for the healthcare system. ${ }^{4-6}$

Hydroxyurea is a medication that can reduce the incidence of pain crises through several pathways, including increasing the amount of fetal hemoglobin in the blood., 2,4-10 From 2002-2014, the National Heart, Lung, and Blood Institute (NHLBI) guidelines recommended hydroxyurea initiation among children and adolescents with specific indications, such as frequent pain episodes, history of acute chest syndrome, severe vasoocclusive events, or severe symptomatic anemia. ${ }^{3}$ However, revised guidelines released in 2014 contain a different recommendation, stating that all children with SCA should be offered hydroxyurea therapy starting at 9 months of age.

Despite the association between hydroxyurea therapy and improved clinical and economic outcomes, center-based and single-state reports of hydroxyurea use among children SAincicate 1 (

with SCA indicate low levels of use; at least half of children that met indication criteria did not receive this medication. ${ }^{8,11-13}$ However, little is known regarding hydroxyurea on a population-level or the extent to which use may vary over time. ${ }^{8,14}$ Therefore, the objective of this multistate study was to describe hydroxyurea use among children with SCA prior to release of the NHLBI revised guidelines. These findings establish a baseline by which to assess potential future changes in hydroxyurea use following the 2014 NHLBI guidelines, as comparable data become available.

\section{Methods}

(1)

Data Souree

Our target population was drawn from the Medicaid programs for the following 6 states with an average to high prevalence of SCA: Florida, Illinois, Louisiana, Michigan, 
South Carolina, and Texas. We selected a convenience sample of states due to completeness of data, availability of validation sources, and prevalence of SCA cases. Medicaid Analytic eXtract (MAX) administrative data were acquired from the Centers for Medicare and Medicaid Services (GMS) for 2005 to 2012; at the time of the study, this was the most recent complete data released by CMS. Administrative data included enrollment history and all paid claims for inpatient, outpatient, emergency department (ED), laboratory, and outpatient pharmacy serviees. Approximately $90 \%$ of children with SCA are enrolled in Medicaid at some point in time and consequently, we expect Medicaid claims data captured a large proportion of the children with SCA in these states. ${ }^{15}$

Study Population

We identified children ages 1 through 17 years with SCA using a case definition of the presence of at least 3 claims for a child within a calendar year that were SCA-related (-) (ICD-9-CM codes 282.61, 282.62). This case definition, targeted to specifically identify children with the subtype SCA, was previously demonstrated to have a high sensitivity (91\%) and specificity ( $80 \%)$ as compared with the gold standard of newborn screening records. ${ }^{16}$ Continuous enrollment in the Medicaid program for at least 1 calendar year within this time period was required; children could contribute multiple, non-sequential time intervals (i.e., 2009 and 2012). We restricted our analysis to children with no other forms of health insurance during the study period to maximize the completeness of Medicaid claims included in this anatysis. Identifying Hydroxyurea Use

To identify all pharmacy claims for hydroxyurea, a list of National Drug Codes (NDCs) for hydroxyurea was generated and verified using RxNorm, a normalized naming system for all drugs produced and maintained by the National Library of Medicine. ${ }^{17}$ For This article is protected by copyright. All rights reserved. 
each person-year in the study population, the total days' supply of hydroxyurea was calculated as the sum of the days' supply of each filled prescription within a calendar year.

During the timeframe of Medicaid data included in this study (2005-2012), hydroxyurea was recommended for children with specific indications. ${ }^{3}$ Therefore, only a subset of children with SCA would have been prescribed hydroxyurea based on this recommendation. We used more than 30 filled days' supply of hydroxyurea as a proxy for an indication for hydroxyurea prescription. More than 30 days' supply is referred to as hydroxyurea use for the remainder of the manuscript. Among children with hydroxyurea use, we assessed the proportion of children with at least 300 days of hydroxyurea. A threshold of 300 days' supply of medication in a calendar year is commonly applied in quality measurement, as it reflects a majority of the year of coverage, without requiring perfect adherence. 16

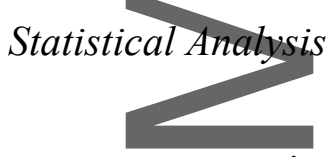

Frequencies and percentages or means and standard deviations (SDs) were determined for all demographics. The total number of days' supply of hydroxyurea for each contributed person-yearwas calculated. Summary statistics of days' supply of hydroxyurea were assessed. Among those with hydroxyurea use (person-years with evidence of more than 30 days' supply), the proportion of children that received at least 300 days' supply was calculated by each year and state. To assess potential predictors of receiving at least 300 days' supply, a logistic regression model with generalized estimating equations to account for children contributing multiple time intervals was conducted among hydroxyurea users. This model estimated the association between potential predictors (age, sex, inpatient visits, outpatient visits, and emergency department visits) and receiving at least 300 days' supply of hydroxyurea, adjusted for year and state. 


\section{Results}

A total of 7963 children with SCA ages 1 through 17 years of age were identified from 2005 to 2012 , contributing a total of 22,424 person-years; less than $1 \%$ were excluded due to other forms of health insurance. The number of person-years varied by state as follows: Florida (6816, 30\%), Louisiana (3753, 17\%), Texas (3727, 17\%), Illinois (3298, 15\%), Michigan $(2708,12 \%)$, and South Carolina (2122, 9\%). Overall, the study population was $48 \%$ femate $(n=3858)$; in 2005 , the average age was 8.8 years $(\mathrm{SD}=5.3)$, which was consistent across each year of observation. The median age was 8 years in Florida, Louisiana, and Texas, and 9 years in Illinois, Michigan, and South Carolina.

Among all person-years, 17,457 (77.9\%) had 0 days of hydroxyurea within a year, $597(2.7 \%)$ had $1-30$ days of hydroxyurea supply within a year, and $4370(19.5 \%)$ were classified as having hydroxyurea use ( $>30$ days of hydroxyurea supply within a year). Among person-years with hydroxyurea use, the average number of annual days' supply was 189 $(\mathrm{SD}=107.7)$. The annual proportion of those with hydroxyurea use who had at least 300 days' supply ranged between 14.9\% (2009) and 19.6\% (2005) (Figure 1).

Among those with hydroxyurea use, having at least 300 days' supply of hydroxyurea was associated with age, inpatient admissions, and outpatient visits. Each increasing year of age and each additional inpatient admission were associated with lower odds. Conversely, more outpatient visits were associated with higher odds of having at least 300 days' supply of hydroxyurea (Table 1).

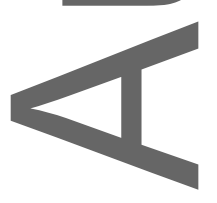




\section{Discussion}

In this multistate analysis, we established a baseline of hydroxyurea use, which can be used to-assess improvements in hydroxyurea use following introduction of the new NHLBI guidelines. Our study indicated that hydroxyurea use among children with SCA was low across all years and states. Among children with evidence of hydroxyurea use, only one in five person-years received more than 300 days of hydroxyurea within a calendar year. This suggests that even among children that use hydroxyurea, medication adherence is low and strategies to increase adherence to hydroxyurea therapy among children with SCA are critically needed. These findings establish a baseline by which to assess potential future improvements in hydroxyurea following the release of the 2014 NHLBI guidelines. ${ }^{7}$

The subsequent impacts of the revised NHLBI pediatric guidelines on hydroxyurea use among children with SCA are not well understood and should be evaluated. For example, the impact of the updated guidelines on physician behavior, such as anticipatory guidance and prescribing behaviors, should be assessed. Changes in caregiver and patient perspective, acceptance, and knowledge regarding hydroxyurea should also be assessed for changes following implementation of the revised guidelines.

This study has several limitations. It is possible that rates of hydroxyurea prescribing by providers to children with SCA are higher than the rates reported here, since medication claims reflect only those prescriptions that were actually filled as opposed to prescribed. In addition, we did not assess which children within the study population met the NHLBI clinical indicators for hydroxyurea use; as a proxy, we included children with more than 30 days' supply as our denominator population. Finally, our results are limited by the accuracy 
and completeness of administrative datasets, which may affect both the identification of the study population as well as measurement of the outcome of interest.

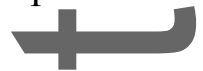

In conclusion, even among children that are already using hydroxyurea, adherence rates are suboptimal. Given the NHLBI updated recommendations that all children with SCA should begin hydroxyurea therapy, it is critical that strategies to increase both initiation and adherence to hydroxyurea be developed and tested among this vulnerable population.

\section{()}

\section{Conflict of Interest Statement}

The authors have no conflicts of interest. No financial disclosures were reported by the authors of this paper.

\section{Reference}

1. CDC. Sickle Cell Disease (SCD): Data \& Statistics. 2016;

https://www.cdc.gov/ncbddd/sicklecell/data.html. Accessed September 1, 2017.

2. $\quad$ National Heart Lung and Blood Institute. Sickle Cell Disease. 2017;

https://Www.nhlbi.nih.gov/health/health-topics/topics/sca. Accessed February 22, 2018

3. National Heart L, and Blood Institute. The Management of Sickle Cell Disease. 2002; https://www.nhlbi.nih.gov/files/docs/guidelines/sc mngt.pdf. Accessed February 22, 2019.

4. Jerrell JM, Tripathi A, McIntyre RS. Prevalence and treatment of depression in children and adolescents with sickle cell disease: a retrospective cohort study. Prim Care Companion CNS Disord. 2011;13(2).

5. CacciottiC, Vaiselbuh S, Romanos-Sirakis E. Pain Management for Sickle Cell Disease in the Pediatric Emergency Department: Medications and Hospitalization Trends. Clin Pediatr (Phila). 2017;56(12):1109-1114.

6. Bou-Maroun LM, Meta F, Hanba CJ, Campbell AD, Yanik GA. An analysis of inpatient pediatric sickle cell disease: Incidence, costs, and outcomes. Pediatr Blood Cancer. 2018;65(1).

7. National Heart Lung and Blood Institute Evidenced-Based Management of Sickle Cell Disease: Expert Panel Report, 2014. 2014; 
https://catalog.nhlbi.nih.gov/sites/default/files/publicationfiles/56-364NFULL.pdf. Accessed 5 April, 2018.

8. Brandow AM, Panepinto JA. Monitoring toxicity, impact, and adherence of hydroxyurea in children with sickle cell disease. Am J Hematol. 2011;86(9):804-806.

9. McGann PT, Ware RE. Hydroxyurea for sickle cell anemia: what have we learned and what questions still remain? Curr Opin Hematol. 2011;18(3):158-165.

10. Thornburg CD, Files BA, Luo Z, et al. Impact of hydroxyurea on clinical events in the BABY HUG trial. Blood. 2012;120(22):4304-4310.

11. Candrilli SD, O'Brien SH, Ware RE, Nahata MC, Seiber EE, Balkrishnan R. Hydroxyurea adherence and associated outcomes among Medicaid enrollees with sickte cell disease. Am J Hematol. 2011;86(3):273-277.

12. Thornburg CD, Calatroni A, Telen M, Kemper AR. Adherence to hydroxyurea therapy in children with sickle cell anemia. J Pediatr. 2010;156(3):415-419.

13. Walsh KE, Cutrona SL, Kavanagh PL, et al. Medication adherence among pediatric patients with sickle cell disease: a systematic review. Pediatrics. 2014;134(6):11751183.

14. Badawy SM, Thompson AA, Penedo FJ, Lai JS, Rychlik K, Liem RI. Barriers to hydroxyurea adherence and health-related quality of life in adolescents and young adults with sickle cell disease. Eur J Haematol. 2017;98(6):608-614.

15. Reeves SL, Jary HK, Gondhi JP, Kleyn M, Wagner AL, Dombkowski KJ.

Pneumococcal vaccination coverage among children with sickle cell anemia, sickle cell trait, and normal hemoglobin. Pediatr Blood Cancer. 2018:e27282.

16. Reeves SL MB, Shevrin CA, McCormick J, Freed GL, Dombkowski KJ. Antibiotic Prophylaxis Among Children with Sickle Cell Anemia. 2017; https://www.qualityforum.org/QPS/3166. Accessed 01/09, 2019.

17. NIH.RxNorm. 2018; https://www.nlm.nih.gov/research/umls/rxnorm/. Accessed May $22,2018$.

Table 1. Multivariable model predicting receipt of at least 300 days of hydroxyurea among children with SCA with evidence of hydroxyurea use ( $\mathrm{N}=4370$ person-years).

\begin{tabular}{lrrr}
\hline Predictor & Odds Ratio & 95\% Confidence Interval & P-Value \\
\hline Inpatient Visits & 0.77 & $0.73,0.81$ & $<0.0001$ \\
ED Visits & 1.01 & $0.99,1.04$ & 0.1982 \\
OutpatientVisits & 1.01 & $1.00,1.02$ & 0.0018 \\
Age & 0.93 & $0.91,0.94$ & $<0.0001$ \\
Sex & & & \\
$\quad$ Male & Reference & Reference & Reference \\
Female & 0.92 & $0.78,1.08$ & 0.2803 \\
State & & & \\
$\quad$ Illinois & Reference & Reference & Reference \\
Michigan & 1.12 & $0.83,1.50$ & 0.4635 \\
Louisiana & 0.93 & $0.71,1.24$ & 0.6339 \\
South Carolina & 0.55 & $0.39,0.79$ & 0.0010 \\
Texas & 0.78 & $0.59,1.03$ & 0.0754
\end{tabular}

This article is protected by copyright. All rights reserved. 


\begin{tabular}{crrr}
$\begin{array}{c}\text { Florida } \\
\text { Year }\end{array}$ & 0.96 & $0.74,1.24$ & 0.7567 \\
2005 & & & \\
2006 & Reference & Reference & Reference \\
2007 & 0.85 & $0.59,1.22$ & 0.3861 \\
2008 & 0.90 & $0.63,1.29$ & 0.5669 \\
2009 & 0.93 & $0.65,1.32$ & 0.6683 \\
2010 & 0.67 & $0.47,0.97$ & 0.0311 \\
2011 & 0.80 & $0.57,1.12$ & 0.1944 \\
2012 & 0.69 & $0.49,0.96$ & 0.0273 \\
\hline & 0.64 & & \\
& & & \\
& & & \\
\end{tabular}

Figure 1. Proportion of children with at least 300 filled days of hydroxyurea among those with hydroxyurea use (greater than 30 days hydroxyurea medication filled)
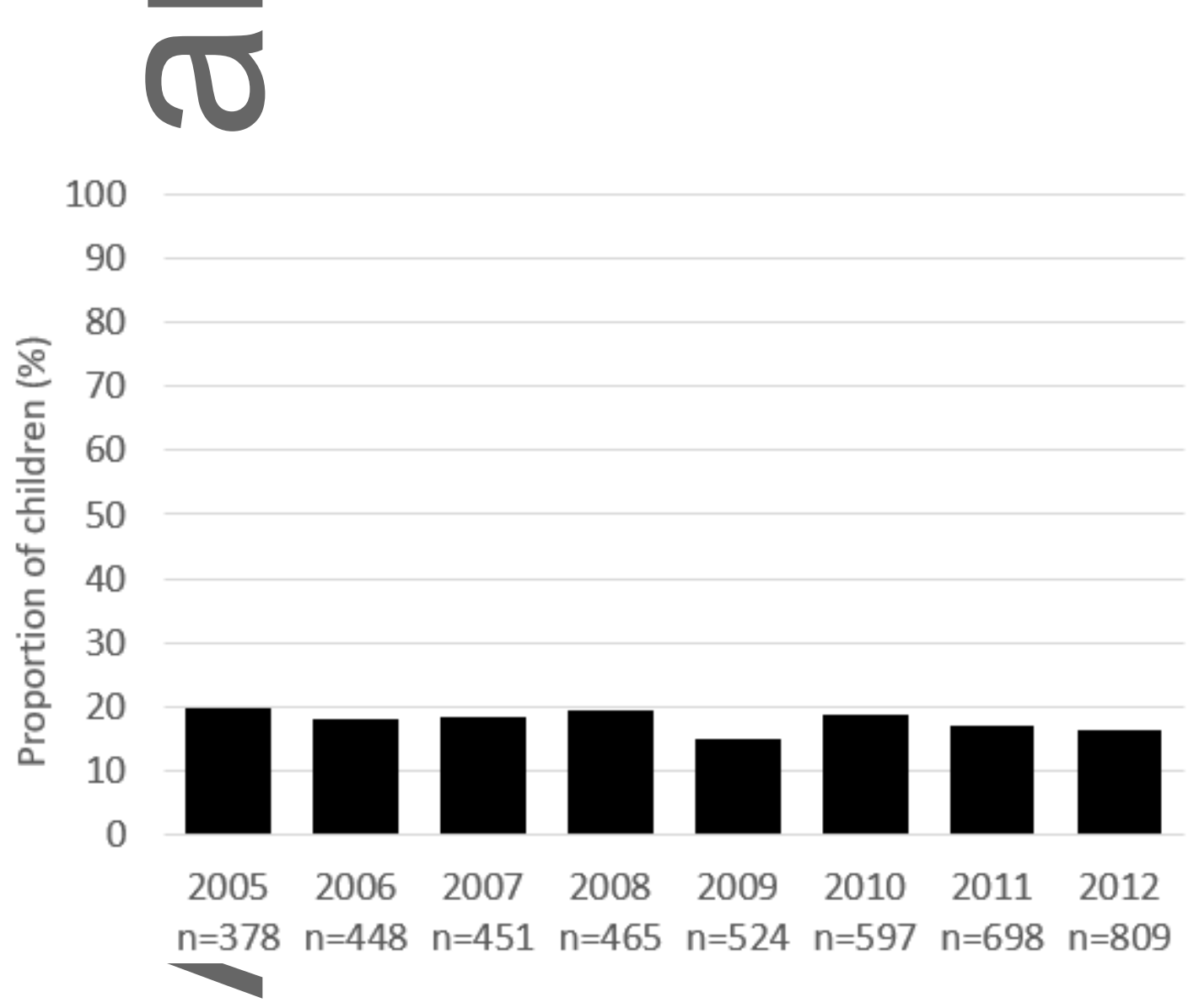